\title{
La participación de los estudiantes en la educación médica
}

\author{
R. Duvivier ${ }^{\mathrm{a}, \mathrm{b}}$, D. Rodríguez-Muñoz ${ }^{\mathrm{a}, \mathrm{c}}$
}

Se presenta una visión general de las diferentes formas en que los estudiantes participan en los debates y decisiones académicas sobre la organización de los estudios de medicina, así como las estructuras organizativas dedicadas a estos objetivos. En el ámbito internacional, los estudiantes de medicina están agrupados en la International Federation of Medical Students' Associations (IFMSA), que mantiene múltiples relaciones con diversas organizaciones del campo de la educación médica con el objetivo de representar la voz de los estudiantes internacionales: Association for Medical Education in Europe (AMEE) y World Federation for Medical Education (WFME). En España, los estudiantes de medicina se agrupan en el Consejo Estatal de Estudiantes de Medicina (CEEM) y la Federación Española de Asociaciones de Estudiantes de Medicina (IFMSA-Spain). Sus actividades se describen en esta colaboración.

Palabras clave. Educación médica. Estudiantes de medicina. Participación de estudiantes.

\section{Student involvement in medical education}

We present an overview of the various ways in which students participate in the debates and academic decisions on the organization of medical studies, and we present institutional structures dedicated to this action. At the international level, medical students are grouped in the International Federation of Medical Students' Associations (IFMSA). It maintains multiple relationships with several organizations in the field of medical education, with the intention of representing the voice of international students: Association for Medical Education in Europe (AMEE), World Federation for Medical Education (WFME). In Spain, medical students are represented in the Consejo Estatal de Estudiantes de Medicina (CEEM) and Feder- ación Española de Asociaciones de Estudiantes de Medicina (IFMSA-Spain). Their activities will be presented in this article.

Key words. Medical education. Participation of students. Students of medicine.

\section{Introducción}

Actualmente, los estudiantes de medicina están fuertemente implicados en su propia educación, ya sea participando en la planificación de currículos, en su evaluación o en la toma de decisiones; desde la institucionalización de la educación médica, la participación de los estudiantes ha sido en todo momento muy importante. Los estudiantes participan en grupos de definición de planes de estudios, comisiones de educación médica y juntas de los centros en los que estudian. De hecho, la participación tiene lugar a todos los niveles posibles de la toma de decisiones, desde el trabajo específico y local hasta juntas de facultad y organizaciones nacionales e internacionales. $\mathrm{Pa}$ rece obvio el porqué. En primer lugar, los estudiantes tienen experiencia de primera mano sobre el currículo y la información que pueden aportar sobre él contribuye decisivamente a su renovación y mejora [1]. En segundo lugar, los estudiantes son los 'consumidores' de la educación y, por tanto, debe escucharse su voz a la hora de preservar la calidad [2]. En tercer lugar, los estudiantes son un colectivo implicado en el desarrollo de la educación médica; por ello, su participación es una legitimación importante en el esfuerzo de enseñanza realizado por una facultad [3]. Por último,

\footnotetext{
International Federation of Medical Students' Associations (IFMSA).

b Estudiante en la Universidad de Maastricht. Países Bajos. Estudiante en la Universidad de Málaga. España.

E-mail dani.rodriguez83@gmail.com
} 
una implicación activa en la toma de decisiones en relación con el currículo es una responsabilidad lógica si es espera que los estudiantes desempeñen un papel activo en el aprendizaje. En resumen: se reconoce ampliamente que la participación de estudiantes y su aportación como miembros activos, competentes y plenamente reconocidos es importante para mejorar la calidad de la educación médica. Sin embargo, a pesar de estos evidentes motivos para favorecer la implicación de los estudiantes, su participación en la toma de decisiones sobre el currículo varía sustancialmente en distintos países, universidades, facultades e incluso dentro de los planes de estudios de una misma facultad.

En este artículo nos centraremos en el currículo y los estudiantes de medicina, y proponemos varios ejemplos de su participación. En segundo lugar, describimos cómo esta participación se organiza internacionalmente y en España, y qué aspectos requieren una atención más detenida e inmediata para su mejora.

\section{Participación de estudiantes en el ámbito internacional}

Internacionalmente, los estudiantes de medicina se integran en la Federación Internacional de Asociaciones de Estudiantes de Medicina -International Federation of Medical Students' Associations (IFMSA)-. IFMSA es una organización de estudiantes de medicina, independiente, no gubernamental y no afiliada a corriente política alguna. Actualmente, cuenta con más de 100 organizaciones miembros de más de 90 países de los seis continentes, y representa a más de 1,2 millones de estudiantes de medicina en todo el mundo. Se fundó en mayo de 1951, está establecida legalmente en los Países Bajos como organización sin ánimo de lucro y está gestionada por y para estudiantes de medicina. Es reconocida oficialmente como organización no gubernamental (ONG) en el marco de las Naciones Unidas y también por la Organización Mundial de la Salud (OMS) como el foro internacional de estudiantes de medicina. Su existencia persigue el objetivo de servir a los estudiantes de medicina en todo el mundo $\mathrm{y}$, a través de ellos, a sus comunidades.

IFMSA trabaja en las siguientes áreas a través de comités permanentes:
- Salud pública.

- Salud reproductiva y sida.

- Derechos humanos y paz.

- Intercambios clínicos.

- Intercambios de investigación.

- Educación médica.

\section{Implicación de IFMSA en la educación médica}

La educación médica debería preocupar a cada estudiante de medicina ya que determina no sólo la calidad de los futuros médicos, sino también de la sanidad desde un punto de vista más amplio. IFMSA tiene un grupo de trabajo dedicado a este ámbito que trabaja para implementar un entorno de aprendizaje óptimo para todos los estudiantes del mundo: el Comité Permanente de Educación Médica (SCOME). Fue uno de los primeros comités de IFMSA desde su fundación en 1951. IFMSA-SCOME sirve como foro de discusión para estudiantes interesados en los diferentes aspectos de la educación médica. Su objetivo es conseguir la excelencia en educación médica en todo el mundo. Para ello, IFMSA en general y SCOME en particular trabajan en tres ámbitos: local, nacional e internacional.

La estrategia más eficaz para conseguir cambios concretos es la local. Más allá incluso de la representación oficial en los órganos de decisión de su facultad, los estudiantes pueden trabajar para generar cambios junto a sus decanos, profesores y otros implicados en el desarrollo de su educación. De esta forma quizá no se consiga cambiar todo el currículo de forma global, pero pequeños cambios en cada una de las distintas asignaturas pueden contribuir de una manera lenta pero también eficaz a la mejora del currículo en su conjunto.

Para ayudar a los miembros locales de IFMSA a promover mejoras en sus propios países y facultades, las principales actividades de SCOME en el ámbito nacional e internacional son las sesiones formativas. Esta formación cubre los aspectos más esenciales de la educación médica como disciplina: la evaluación, las metodologías de enseñanza y aprendizaje, la estructura y diseño de currículos, etc. Otras tareas de SCOME son la realización de jornadas, conferencias y talleres centrados en aspectos específicos, promover y fa- 
cilitar el diálogo entre estudiantes y facultades, participar en evaluaciones externas sobre docencia, recopilar las opiniones de estudiantes de medicina en el ámbito local e intentar implementarlas, y representar a estudiantes ante los órganos de decisión relevantes.

Los miembros de SCOME, además, mantienen un contacto constante, compartiendo experiencias y ejemplos de su trabajo en este campo, a través de foros, listas de correo electrónico y una plataforma basada en tecnología 'wiki', diseñada especialmente para ello: la 'SCOME Wikipedia' (www.ifmsa.org/scome/wiki). Aquí, los estudiantes pueden compartir y encontrar información sobre los sistemas educativos en medicina en los diferentes países, sobre proyectos específicos, etc., tanto actuales como de un amplio archivo de recursos utilizados en el pasado.

Además, dos veces al año, aproximadamente 800 estudiantes de cerca de 100 países se reúnen para compartir su experiencia e incrementar su formación durante las asambleas generales. Además, se organizan reuniones regionales en los períodos interasamblearios para mantener a los estudiantes informados e implicados. Los resultados de estas reuniones se comparten con todos los miembros, así como con el público general, a través de la 'SCOME Wikipedia'. Además, el objetivo es publicar nuestro punto de vista en determinados temas a través de declaraciones y posicionamientos.

En los últimos años hemos dedicado una parte importante de nuestro trabajo a los cambios que implicaba el nuevo Espacio Europeo de Educación Superior y el proceso de Bolonia. Esto ha producido una serie de trabajos que se han publicado en revistas científicas como Medical Teacher o Medical Education [4-7]. Un ejemplo, que resultó de gran utilidad en España y en diversos foros, ha sido el European Core Curriculum $[8,9]$. En respuesta a los cambios propuestos en la educación superior en Europa, los estudiantes de medicina se pusieron de acuerdo en una serie de resultados de aprendizaje que todos los médicos deberían haber alcanzado para graduarse. Estos resultados de aprendizaje esenciales comunes constituirían el Core Curriculum europeo de acuerdo con las regulaciones europeas relevantes. Este documento expresa la opinión de los estudiantes de medicina sobre qué habilidades, conocimientos y actitudes deberían adquirir los licenciados en medicina en facultades europeas y, por tanto, qué competencias deberían evaluarse. El
Core Curriculum detalla 76 resultados del aprendizaje estructurados en nueve dominios: habilidades clínicas, comunicación, razonamiento crítico, salud pública/social, aprendizaje a lo largo de la vida, profesionalismo (actitudes, responsabilidades y desarrollo personal), enseñanza, trabajo en equipo y conocimientos teóricos. Durante años hemos sugerido el empleo de este currículo como un marco fácilmente adaptable y ajustable a las necesidades nacionales y locales de formación, sirviendo como base común con el objetivo de mantener e incluso mejorar la calidad de la educación médica, la sanidad y la movilidad en Europa. La necesidad de un currículo central común es una opinión ampliamente compartida por otras organizaciones e instituciones en Europa, con iniciativas como el Tuning Project for Medicine [10] y, en España, con la reciente publicación de El médico del futuro, por la Fundación Educación Médica [11], o la exhaustiva definición de los objetivos de aprendizaje en el Libro blanco: título de grado en Medicina [12], elaborado por la Conferencia Nacional de Decanos de Facultades de Medicina españolas.

\section{Nuestro trabajo en el ámbito internacional}

IFMSA colabora con organizaciones en el campo de la educación médica para representar la voz de los estudiantes internacionalmente. El objetivo es mantener un flujo de comunicación constante entre los ámbitos local, nacional e internacional. Actualmente, IFMSA está representada en la junta directiva de la AMEE (Association for Medical Education in Europe) y en el consejo de la Federación Mundial de Educación Médica (WFME), además de trabajar con sus seis organizaciones regionales.

\section{Implicación de los estudiantes de medicina en educación médica en España}

El trabajo de los estudiantes de medicina en relación con la educación médica en España ha variado de forma importante en los últimos años. Este trabajo ha pasado de centrarse principalmente en actividades a corto plazo, basadas en proyectos 
concretos con el objetivo de suplir carencias formativas en los currículos, a prestar también, actualmente, una atención preferente a una visión más amplia de la educación médica encaminada a aprovechar el amplio rango de posibilidades de promover cambios y mejoras a través de otros medios. Este cambio se ha canalizado a través de las dos principales organizaciones de estudiantes de medicina en España: el Consejo Estatal de Estudiantes de Medicina (CEEM) y la Federación Española de Asociaciones de Estudiantes de Medicina (IFMSA-Spain). Ambas organizaciones han venido mostrando interés e implicación en la educación médica desde estadios iniciales de su desarrollo, dedicando una parte importante de sus recursos a formar a sus miembros para emplear esa fuerza como generador de cambio y mejora.

Localmente, ambas organizaciones han tenido un impacto directo y fácilmente cuantificable en el desarrollo, por parte de los estudiantes, de nuevas habilidades y competencias a través de los proyectos anteriormente mencionados. Muchos de estos proyectos se han desarrollado gracias a la estrecha colaboración con profesores y otros miembros de sus facultades, cuyo apoyo ha sido crucial para la puesta en marcha de cursos de inglés médico, de comunicación médico-paciente, de suturas, de atención en emergencias y reanimación cardiopulmonar, etc. Estos cursos, organizados en respuesta a necesidades percibidas o demandas explícitas de estudiantes de medicina por ciertas carencias en su formación, con frecuencia han supuesto el primer paso hacia la implementación de muchas de esas oportunidades de aprendizaje como parte del currículo. En estos procesos, todos los estudiantes de medicina se han beneficiado de un sólido entendimiento entre estudiantes y facultad. Por una parte, se ha demostrado el enorme valor de las organizaciones de estudiantes de medicina por su capacidad de detectar defectos o carencias en la educación médica, así como por su flexibilidad y rapidez a la hora de responder a esas carencias con alternativas de formación. Asimismo, las facultades de medicina y sus miembros han empleado esas capacidades de los estudiantes para consolidar tales iniciativas y convertirlas en mejoras concretas en los planes de estudios. Otros proyectos que se han desarrollado en los últimos años han aportado nuevas oportunidades a los estudiantes de medicina, con iniciativas como el trabajo conjunto con las sociedades de medicina de familia SEMERGEN y SEMFyC, para facilitar el acceso a sus cursos de formación, o el diseño y desarrollo de cuestionarios para evaluar la calidad de la docencia y de los métodos de evaluación en las facultades de medicina.

Tanto en el ámbito local como nacional hemos experimentado también una importante mejora en la representatividad de los estudiantes, donde el CEEM ha alcanzado el máximo grado de profesionalismo en su historia tras una extensa reforma y un intenso trabajo para garantizar su legitimidad a todos los niveles. Esto, junto con el desarrollo de acuerdos más amplios con IFMSA-Spain que han enriquecido a ambas organizaciones tanto en sus puntos de vista como en número de miembros, ha generado una mejora de la participación de los estudiantes en las juntas de gobierno de sus facultades y en importantes movilizaciones de estudiantes de medicina en el ámbito nacional que han arrojado importantes resultados, como el reconocimiento de los estudios de medicina como grado y máster en España, que permanecía como excepción en el Espacio Europeo de Educación Superior hasta hace unos meses.

Recientemente, los estudiantes de medicina en España se han implicado en mayores retos de ámbito europeo y mundial. Numerosos miembros del CEEM y de IFMSA-Spain participaron activamente en la organización y el desarrollo de importantes eventos y reuniones, como la conferencia anual de la AMEE y el XIX Congreso de la Sociedad Española de Educación Médica (SEDEM) en Málaga, o la organización del simposio europeo anual sobre el proceso de Bolonia de IFMSA en Córdoba, todos ellos en el año 2009.

Sin embargo, y a pesar de los éxitos conseguidos recientemente a través de este trabajo o de la colaboración con otras organizaciones e instituciones, continúa habiendo aspectos preocupantes que afectan negativamente a la capacidad de los estudiantes de medicina de implicarse y trabajar en educación médica en nuestro país, y la responsabilidad de dejar atrás esos obstáculos es compartida por los decanos y juntas de facultad, profesores, organizaciones e instituciones dedicadas a la educación médica y los propios estudiantes. Es responsabilidad de los decanos, profesores y juntas de las facultades asumir que la implicación de los estudiantes en cuestiones referentes al currículo y a su educación es esencial. Para conseguirlo, el papel de decanos y juntas no debe ser el de esperar a escuchar lo que los estudiantes tengan que decir, sino el de buscar activamente su 
implicación y apoyar su formación y el crecimiento de las asociaciones locales que los sustentan. Debemos ser conscientes de que la carrera de medicina es sumamente exigente, y que aquellos estudiantes que deciden dedicar una parte de su tiempo a mejorar su educación y la de sus compañeros, futuros médicos, deben encontrar en los responsables de sus facultades ayuda y apoyo en lo posible, y no obstáculos. La idea tradicional de que el único deber de los estudiantes es el de estudiar, aprender y aprobar sus asignaturas debe dejarse atrás, especialmente a la luz de las numerosas experiencias que muestran que la implicación de los estudiantes en la mejora de su educación es beneficiosa para sí mismos, a través del desarrollo de importantes habilidades, y para sus compañeros estudiantes de medicina. En España, la Conferencia Nacional de Decanos y la SEDEM pueden desempeñar un papel muy importante en liderar este cambio de mentalidad, aún muy necesario en algunas facultades.

Por otra parte, las organizaciones de estudiantes como IFMSA-Spain y el CEEM deben estar a la altura del papel que reclaman y responder a las oportunidades que se les presentan con un trabajo sólido, profesionalismo y responsabilidad, manteniendo presente que sus éxitos y fracasos influyen decisivamente no sólo en sus líderes y sus miembros activos, sino en todos los estudiantes de medicina.

En resumen, la situación actual de la implicación de los estudiantes de medicina en España en la educación médica ha mejorado de manera importante en los últimos años. Las organizaciones de estudiantes de medicina, sus líderes y todos sus miembros, así como todos los que han estado dispuestos a escucharlos y a trabajar junto a ellos, deben felicitarse por ese éxito que es, al fin y al cabo, un éxito que se extiende a nuestra sociedad a través de la mejora de sus médicos, y con ello la mejora de su atención sanitaria. Pero aún hay mucho margen de mejora, numerosas cuestiones que necesitan atención y una idea esencial que debe ser asumida por todos los implicados, de una $\mathrm{u}$ otra forma, en la educación médica: que cada uno de nosotros tiene una responsabilidad social hacia producir los mejores médicos posibles, y que todo aspecto de la formación de un médico que se deja en segundo plano, se devalúa. Es, por tanto, una responsabilidad tanto de los estudiantes como de los profesionales de la educación médica el hecho de cuestionarnos constantemente si estamos consiguiendo lo que nos proponemos como meta, $\mathrm{y}$ si las metas que nos marcamos son las correctas.

\section{Bibliografía}

1. Slater C. Student participation in curriculum planning and evaluation. J Med Educ 1969; 44: 67-8.

2. Visser K, Prince KJ, Scherpbier AJ, Van der Vleuten CP, Verwijnen GM. Students can be full partners in designing their education. Acad Med 1997; 72: 1034-5.

3. Sallis E. Total quality management in education. London: Kegan; 1996.

4. Duvivier RJ, Hilgers J, Davaris N, Rodríguez-Muñoz D. Implementation of the Bologna two-cycle system in medical education -the students' view. Med Teach 2009; 31: 376-7.

5. Duvivier RJ, Brouwer EE, Weggemans M. Medical education in global health: student initiatives in the Netherlands. Med Educ 2010; 44: 527-30.

6. Duvivier RJ, Weggemans M. Medical education in Europe: a commentary from students. Tijdschrift voor Medisch Onderwijs 2010; 9: 220-1.

7. Duvivier RJ, Weggemans M. Joint issue TMO/ZMA: reply from international medical students GMS. Z Med Ausbild 2010; 27: Doc40.

8 IFMSA, EMSA, Hilgers J, De Roos P. European core curriculum: the students' perspective, Bristol, UK, 10 July 2006. Med Teach 2007; 29: 270-5.

9. URL:http://wiki.ifmsa.org/scome/index.php?title=european _core_curriculum_-_the_students\%27_perspective. [30.10.2010].

10. Cumming A, Ross M. The Tuning Project for medicine -learning outcomes for undergraduate medical education in Europe. Med Teach 2009; 29: 636-41.

11. El médico del futuro. Barcelona: Fundación Educación Médica; 2009.

12. Libro blanco: título de grado en Medicina. URL: www. sedem.org/libroblanco.pdf. [30.10.2010]. 